\title{
A Clinico-Hematological Study of Cases of Leucoerythroblastic Reaction
}

\author{
Tejaswini Waghmare, Daksha Prabhat* and Naveen Vairamoorthy D. \\ Department of Pathology, Seth GS Medical College and KEM Hospital, Mumbai, India
}

\begin{abstract}
Background: Leucoerythroblastic Reaction (LER) is defined as the presence of immature red cells and immature white cells of the myeloid series in peripheral blood. Literature has revealed various causes for LER, which include hemolytic anemia, infections, megaloblastic anemia, liver diseases and others.

Objectives: To study the hematological changes, especially peripheral blood smear (PBS) findings in LER cases.

Methods: A total number of 100 consecutive cases which revealed LER on peripheral blood smear (PBS) were included in this study. Various hematological parameters such as hemoglobin, total leucocyte count, platelet count as obtained from the automated blood cell counter and PBS findings were analysed.

Results: In the present study, majority of the cases (30\%) were in the age range of 21 to 30 years, with equal gender distribution. Anemia was seen in $87 \%$ of the cases, leucocytosis in $79 \%$ and thrombocytopenia in $60 \%$. Polychromasia with increased reticulocytes was noted in $55 \%$ of the cases. Nucleated RBCs were noted in all cases. All cases showed shift to left in the myeloid series. Commonest cause of LER encountered was hemolytic disease (29\%) followed by liver diseases $(22 \%)$ and septicemia (18\%).
\end{abstract}

Conclusion: The present study emphasizes various etiologies of LER and the importance of reporting LER in PBS.Examination of peripheral blood smear for morphology of RBCs, WBCs and platelets gives a clue to the etiology.

\section{Keywords: Hematology, Leucoerythroblastic Reaction, Peripheral Blood Smear}

\section{Introduction}

Leucoerythroblastic reaction (LER) is defined as 'the presence in the peripheral blood, of the immature red cells and few immature white cells of the myeloid series'. ${ }^{[1]}$ While LER was known to be classically associated with bone marrow infiltrations and disseminated malignancy, studies in literature revealed various other causes to be associated with its occurrence. ${ }^{[2,3]}$ Notable causes among them are as follow

1. Hemolytic diseases including congenital and acquired hemolytic anemias

2. Bone marrow infiltrations by carcinomatosis, leukemias, lymphomas, Hodgkin's disease and in myelofibrosis and myelomatosis

3. Bacterial infections including cellulitis, peritonitis, abscess and wound sepsis

4. Liver diseases including fulminant hepatitis and cirrhosis

5. Megaloblastic anemia

6. Miscellaneous causes including drug reaction, bleeding and uremia ${ }^{[1]}$

The incidence of LER is found to be around 0.33 to $0.45 \%$. ${ }^{[4]}$
Literature suggests that though LER is present in a variety of clinical conditions, there is a mechanism common to most of these - the presence of stress or damage to the marrow and the evolution of areas of extramedullary hematopoiesis, where a well organised sinusoidal structure may not exist and the physiologic and physical barriers restraining immature cells may not be effective as in the marrow. ${ }^{[5]}$ Leucoerythroblastosis can be associated with various causes but bone marrow examination is essential for differential diagnosis with leukemia. ${ }^{[6]}$

The morphology of the circulating cells may offer clues as to the cause of the leucoerythroblastic picture.

With this backdrop, the present study was planned to study the hematological changes, especially peripheral blood smear (PBS) findings in LER cases.

\section{Materials and methods}

Ethics: Prior approval was taken from Institutional Ethics Committee before commencing the study. A written informed consent was taken from patients or guardian/ parents as applicable.

Methodology: The present study was an observational, cross-sectional study conducted over two years of duration 
in the Department of Pathology of 'a' tertiary care hospital in Mumbai. A total of 100 cases of leucoerythroblastic reaction detected on peripheral blood smear from all the age groups and either gender, except for neonates were included in the study.

Samples which revealed leucoerythroblastic picture on peripheral blood smear among the ones received in the hematology and clinical pathology section (which processes samples of hospitalized patients) of the pathology department for routine hematological investigation, i.e., complete blood count were reviewed. The values of $\mathrm{Hb}$, RBC indices, total leucocyte count, platelet count of these already processed samples (on automated blood cell counter) were noted and the peripheral blood smears made from these same samples were observed for morphology of RBCs, WBCs and platelets. The diagnosis of leucoerythroblastic reaction was based on Vaughan's original definition ${ }^{[1]}$ i.e., the coexistence of myeloid precursors (metamyelocytes, myelocytes, promyelocytes and myeloblasts) and erythroid precursors in the peripheral blood.

The patient information was collected in a preformed structured proforma. The clinical details were obtained from the medical records and other specialized hematological investigations, such as bone marrow examination reports were obtained, wherever available.

Detailed study of morphology of RBCs, WBCs and platelets as observed in peripheral blood smear was performed and the same was correlated with other hematological parameters.

Statistical Analysis: All the categorical and quantitative data was presented as frequency and proportion. Analysis of data was done using Microsoft Excel® 2010.

\section{Results}

i). Demographics of Cases: Of all the cases we received over two years, only $0.15 \%$ cases had evidence of leucoerythroblastic reaction. We analysed 100 consecutive cases showing LER on PBS. Of these 100,50 were females. 30 cases were seen in the age group of 21-30 years followed by 17 cases from 31-40 years and least being from 71-80 years and infants, five each. Demographics are depicted in the figure 1.

ii) Distribution of Hemoglobin [Hb] Across Cases: Out of 100 cases, 87 cases had hemoglobin $(\mathrm{Hb})$ less than $10 \mathrm{gm} \%$. Nine cases had $\mathrm{Hb}$ less than $4 \mathrm{gm} \%$ while one had $\mathrm{Hb}$ above $12 \mathrm{gm} \%$. Distribution of $\mathrm{Hb}$ across cases is in table 1 .

iii) Total Leukocyte Count [TLC] Distribution: Two patients had TLC of $<4000 / \mathrm{cmm}$ while 19 patients had normal TLC (4000/cmm - 11000/cmm). Remaining patients had count more than $11000 / \mathrm{cmm}$, out of which nine patients had TLC count $>40000 / \mathrm{cmm}$.

iv) Platelet Count Distribution: Thrombocytopenia (platelet count less than $1.5 \mathrm{lakh} / \mathrm{cmm}$ ) was seen with 60 patients. While eight patient had high platelet counts (more than four lakh/cmm).

v) Distribution of Causes: In the present study, hemolytic diseases (HD) was the most common (29\%) cause of the leucoerythroblastosis, followed by liver diseases (LD) $(22 \%)$ and septicemia (SP) (18\%). Megaloblastic anemia (MA) was diagnosed in 11 patients, eight were diagnosed as myeloproliferative disorders (MPD) while ten as miscellaneous (MIS) where cause could not be classified. Metastatic carcinoma (MCa) and multiple myeloma (MM) was diagnosed in one patient each. Distribution of etiology of patients with LER is given in Figure 2.

Among 29 cases of hemolytic diseases, there were nine cases of thalassemia major [Fig 3], three cases of thalassemia intermedia, one case of thalassemia trait, seven cases of sickle cell anemia, two cases of sickle beta thalassemia, five cases of autoimmune hemolytic anemia, one case of hemolysis secondary to prosthetic valve and one case of hemolysis following snake bite.

Among 22 cases of liver diseases, commonest cause was alcoholic liver disease (5), followed by viral hepatitis (4) and obstetrics related liver disease (3). This was followed by cases of Wilson's disease (2), dengue related liver disease (2), AKT induced liver diseases (2) and other causes.

Among the 18 cases of septicemia - abscesses (of liver or spleen) were seen in three cases, three cases had respiratory tract infection, two cases had cellulitis of limb, two had spontaneous bacterial peritonitis, two had post-operative sepsis and two cases were of puerperal sepsis.

Among 11 cases of megaloblastic anemia, six were deficient only in vitamin B12, three were deficient in both vitamin B12 and folic acid and remaining two were due to folic acid deficiency secondary to drugs.

Among the eight cases of myeloproliferative disorders there were four cases each of chronic myeloid leukemia and myelofibrosis [Fig 4,5].

vi) Hemoglobin distribution among various causes of LER: Cases with low hemoglobin levels were commonly seen with megaloblastic anemia, followed by hemolytic diseases and liver diseases. One case of autoimmune vasculitis (classified under miscellaneous) had the highest $\mathrm{Hb}$ level [12.6 gm\%] 
in our study. Distribution of $\mathrm{Hb}$ levels among various causes of LER is given in table 2.

vii) Total leucocyte count distribution among various causes of LER: Maximum number of cases (10) with normal count (between $4000 / \mathrm{cmm}$ and $11000 / \mathrm{cmm}$ ) were seen with megaloblastic anemia. One case of megaloblastic anemia had low count (less than 4000/ $\mathrm{cmm}$ ). Three out of eight cases with myeloproliferative disorders showed corrected total leucocyte count more than $40000 / \mathrm{cmm}$. Maximum number of cases with total count between $25000 / \mathrm{cmm}$ and $40000 / \mathrm{cmm}$ were seen with septicemia. Distribution of TLC among various causes of LER in presented in table 3.

viii) Platelet count distribution among various causes of LER: Lower platelet counts (less than $1.5 \mathrm{lakh} / \mathrm{cmm}$ ) were commonly encountered with megaloblastic anemia, followed by septicemia and liver diseases. Instances of normal and high platelet counts were seen commonly with hemolytic diseases. Platelet count distribution among various causes of LER is given in table 4 .

ix) Average values of $\mathrm{Hb}$, TLC and platelet count among various causes of LER: In the present study, average hemoglobin level was lowest with megaloblastic anemia, followed by hemolytic diseases. Higher average hemoglobin values were seen with septicemia. Highest average corrected total leucocyte count was seen with myeloproliferative disorders, followed by septicemia and liver diseases. Lower total leucocyte counts were commonly associated with megaloblastic anemia. Highest average platelet values were associated with myeloproliferative disorders, followed by hemolytic diseases. Lowest average platelet values were seen with megaloblastic anemia, followed by septicemia. Megaloblastic anemia had the lowest average values of all the above 3 parameters. Average values of $\mathrm{Hb}$, TLC and platelet count among various causes of LER are presented in table 5.

x) Distribution of nucleated RBCs (nRBCs), Reticulocyte count and WBC precursors among various causes of LER: In the present study, nRBC counts were in the range of 2 to 588 with an average of 43 per 100 WBCs counted. Reticulocyte counts varied from 1 to 33 percentage, with an average of $5.5 \%$. Around 55\% of the cases showed increased reticulocyte count (more than $2.5 \%$ ). This corresponded to the number of cases showing polychromasia on PBS. Counts of leucocyte (myeloid) precursors (metamyelocytes, myelocytes, promyelocytes and myeloblasts) varied from 3 to 44 percentage, with an average of 11 .
Higher nucleated RBC counts and high average nucleated $\mathrm{RBC}$ count were seen with hemolytic diseases. Lower average $n R B C$ counts were seen with megaloblastic anemia. Reticulocyte counts were highest in hemolytic diseases (as high as 33\%) and also was average high reticulocyte count $(9 \%)$. This was followed by megaloblastic anemia which had an average reticulocyte count of $8 \%$. Highest number and high average number of WBC precursors were seen with myeloproliferative diseases and this was followed by septicemia. Higher number of band forms were seen in cases of septicemia. PBS in a case of multiple myeloma showing shift to left in myeloid series and a plasma cell [inset] in fig 6. PBS picture of a case of metastatic carcinoma showing LER is depicted in fig 7 and the bone marrow of the same case showing malignant epithelial cells in Fig. 8. Distribution of nRBCs, reticulocyte counts and percentage of WBC precursors among various causes of LER is given in table 6.

xi) Mean corpuscular volume (MCV) and Red blood cell distribution width (RDW-SD) among various causes of LER: In the present study, MCV values ranged from 53.2 to $124 \mathrm{fL}$, with an average of $85 \mathrm{fL}$. RDW - SD values ranged from 11.3 to 40 , with an average of 23 . Higher values of MCV were seen with megaloblastic anemia, highest MCV noted being $124.2 \mathrm{fL}$, with an average of $103 \mathrm{fL}$. Average low MCV values were seen with hemolytic diseases. Higher values of RDW - SD were seen with myeloproliferative disorders, followed by hemolytic diseases. Distribution of MCV and RDW - SD values among various causes of LER is given in table 7 .

\section{Discussion}

i) Demographics: In present study, the incidence of LER was $0.15 \%$ among all the blood samples received in the hematology laboratory. This is lower than the incidence reported in the study by Ken Sang Lee et al. $[0.33 \%]$ and by Retief et al. $[0.45 \%] .{ }^{[4,7]}$ This difference can be due to the relative homogeneity of the population as the study is conducted at one centre only.

In current study we had equal distribution of cases across the gender. This was found contrasting with the findings by Retief et al. [70 females, 30 males] while it is comparable with the findings by Ken Sang Lee et al. [57 males, 43 females]. ${ }^{[4,7]}$

In the present study, majority of the patients were in third decade (30\%), followed by fourth decade (17\%) and seventh decade (12\%). 12\% of the patients belonged to pediatric age group (12 years or less). These results were comparable to those by Retief et al.[majority of 
Table 1. Distribution of $\mathrm{Hb}$ across cases.

\begin{tabular}{|c|c|}
\hline Hb (in gm \%) & Number of cases \\
\hline$<4$ & 9 \\
\hline 4 to 10 & 78 \\
\hline 10 to 12 & 12 \\
\hline$>12$ & 1 \\
\hline
\end{tabular}

Table 2. Distribution of Hb levels among various causes of LER.

\begin{tabular}{|c|c|c|c|c|c|c|c|c|}
\hline \multicolumn{10}{|c|}{ Hb level distribution among various causes } \\
\hline & HD & LD & SP & MA & MPD & MCa & MM & MIS \\
\hline$<4$ & 5 & 2 & 0 & 3 & 1 & 0 & 0 & 0 \\
\hline $4.1-6$ & 7 & 2 & 2 & 5 & 1 & 0 & 1 & 5 \\
\hline $6.1-8$ & 8 & 11 & 5 & 2 & 3 & 0 & 0 & 1 \\
\hline $8.1-10$ & 8 & 6 & 6 & 1 & 1 & 0 & 0 & 1 \\
\hline $10.1-12$ & 1 & 1 & 5 & 0 & 2 & 1 & 0 & 2 \\
\hline$>12$ & 0 & 0 & 0 & 0 & 0 & 0 & 0 & 1 \\
\hline & 29 & 22 & 18 & 11 & 8 & 1 & 1 & 10 \\
\hline
\end{tabular}

Table 3. Distribution of TLC among various causes of LER.

\begin{tabular}{|c|c|c|c|c|c|c|c|c|c|}
\hline \multicolumn{9}{|c|}{ Distribution of TLC among various causes of LER } \\
\hline & HD & LD & SP & MA & MPD & MCa & MM & MIS \\
\hline$<4000$ & 1 & 0 & 0 & 1 & 0 & 0 & 0 & 0 \\
\hline $4000-11000$ & 3 & 3 & 2 & 10 & 0 & 0 & 0 & 1 \\
\hline $11000-25000$ & 22 & 12 & 8 & 0 & 3 & 1 & 1 & 0 \\
\hline $25000-40000$ & 2 & 5 & 6 & 0 & 2 & 0 & 0 \\
\hline$>40000$ & 1 & 2 & 2 & 0 & 3 & 0 & 0 & 1 \\
\hline & 29 & 22 & 18 & 11 & 8 & 1 & 1 & 10 \\
\hline
\end{tabular}

Table 4. Platelet count distribution among various causes of LER

\begin{tabular}{|c|c|c|c|c|c|c|c|c|}
\hline \multicolumn{7}{|c|}{ Platelet count distribution among various causes of LER } \\
\hline & HD & LD & SP & MA & MPD & MCa & MM & MIS \\
\hline$<15000$ & 0 & 0 & 1 & 1 & 0 & 0 & 0 & 0 \\
\hline 15000-1 lakh & 8 & 11 & 10 & 8 & 3 & 1 & 0 & 4 \\
\hline 1 lakh-1.5 lakh & 4 & 4 & 3 & 1 & 1 & 0 & 0 & 0 \\
\hline 1.5 lakh -4 lakh & 12 & 6 & 3 & 1 & 3 & 0 & 1 & 6 \\
\hline >4 lakh & 5 & 1 & 1 & 0 & 1 & 0 & 0 & 0 \\
\hline & 29 & 22 & 18 & 11 & 8 & 1 & 1 & 10 \\
\hline
\end{tabular}

Table 5. Average values of Hb, TLC and platelet count among various causes of LER.

\begin{tabular}{|c|c|c|c|c|c|c|c|c|}
\hline \multicolumn{8}{|c|}{ Average values of Hb, TLC and platelet count among various causes of LER } \\
\hline & HD & LD & SP & MA & MPD & MIS & MCa & MM \\
\hline Hemoglobin (gm\%) & 6.5 & 7.2 & 8.5 & 5.5 & 7.1 & 7.8 & 10.9 & 5.8 \\
\hline TLC (/cmm) & 16400 & 22250 & 27000 & 7500 & 34000 & 18500 & 13600 & 16800 \\
\hline Platelet count (Lakhs/cmm) & 2.5 & 1.5 & 1.3 & 0.73 & 3.5 & 2.1 & 0.37 & 1.5 \\
\hline
\end{tabular}

Table 6. Distribution of $\mathrm{nRBC}$, reticulocyte counts and percentage of WBC precursors among various causes of LER

\begin{tabular}{|c|c|c|c|}
\hline \multicolumn{2}{|c|}{ Distribution of nRBCs and reticulocyte counts and percentage of WBC precursors among various causes of LER } \\
\hline Hemolytic diseases & nRBCs (average) & Reticulocyte (average) & WBC Precursors (average) \\
\hline Liver diseases & $4-588(106)$ & $2-33(9)$ & $3-21(8)$ \\
\hline Septicemia & $2-72(18)$ & $1-10(3.5)$ & $3-23(10)$ \\
\hline Megaloblastic anemia & $2-85(17)$ & $1-9(2.5)$ & $4-30(11)$ \\
\hline
\end{tabular}




\begin{tabular}{|c|c|c|c|}
\hline \multicolumn{4}{|c|}{ Distribution of $\mathrm{nRBCs}$ and reticulocyte counts and percentage of WBC precursors among various causes of LER } \\
\hline Myeloproliferative disorders & nRBCs (average) & Reticulocyte (average) & WBC Precursors (average) \\
\hline Miscellaneous & $3-72(20)$ & $1-6(2.5)$ & $4-44(25)$ \\
\hline Overall & $2-37(15)$ & $1-11(5)$ & $4-21(10)$ \\
\hline
\end{tabular}

Table 7. Distribution of MCV and RDW - SD values among various causes of LER.

\begin{tabular}{|c|c|c|}
\hline \multicolumn{2}{|c|}{ Distribution of MCV and RDW - SD values among various causes of LER } \\
\hline & MCV fL (average) & RDW -SD (average) \\
\hline Hemolytic anemia & $62.3-120.1(79)$ & $16.6-35(24.5)$ \\
\hline Liver diseases & $58-99.3(85)$ & $13.4-30.2(19.7)$ \\
\hline Septicemia & $63.4-88.7(82.7)$ & $13.2-39(25)$ \\
\hline Megaloblastic anemia & $79.7-124.2(103)$ & $19.1-36.5(27.1)$ \\
\hline Myeloproliferative disorders & $53.2-94.8(78.4)$ & $13.6-24.9(18.1)$ \\
\hline Miscellaneous & $77.4-103.5(90.5)$ & $11.3-40(23)$ \\
\hline
\end{tabular}

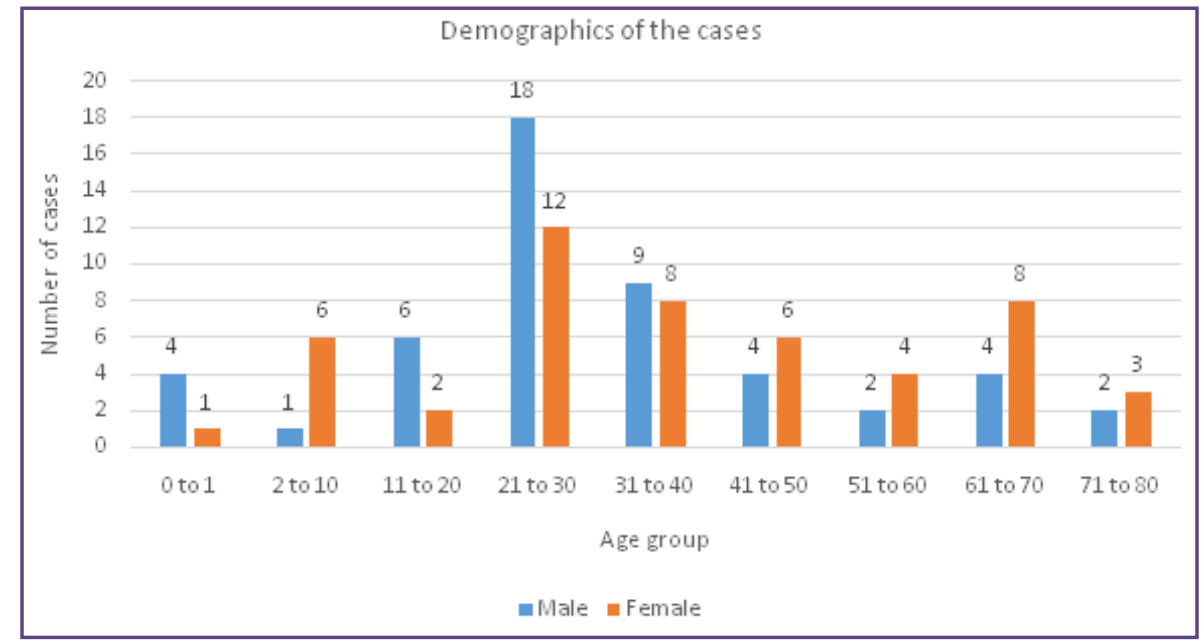

Fig. 1: Demographics of cases.

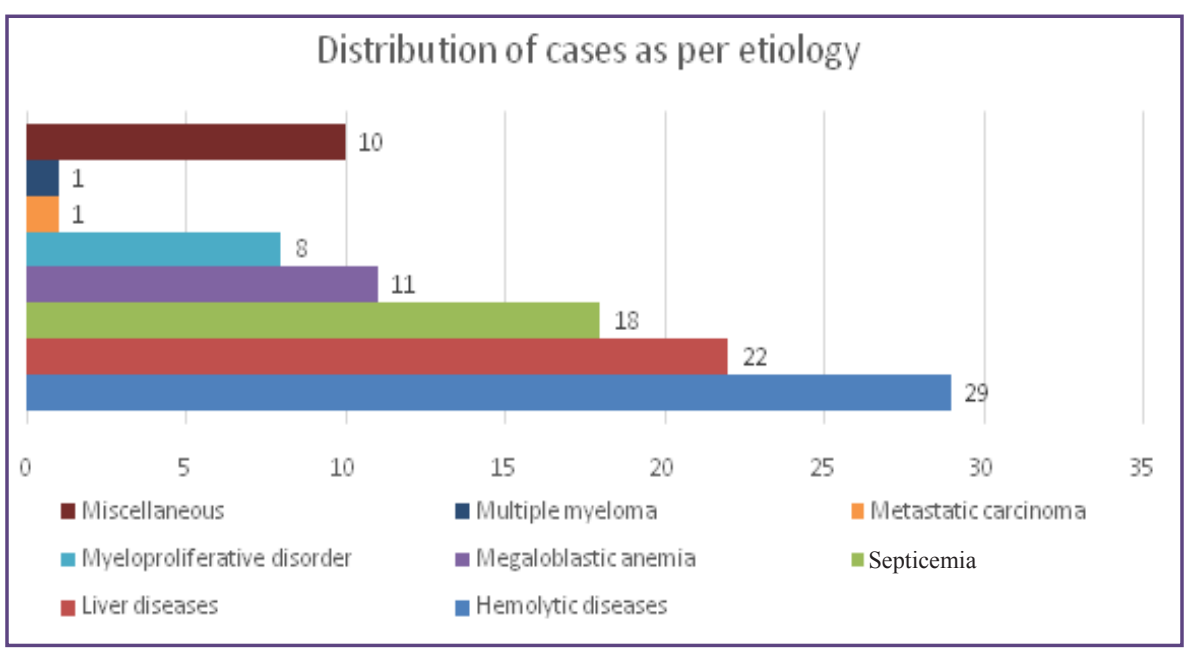

Fig. 2: Distribution of cases as per etiology. 


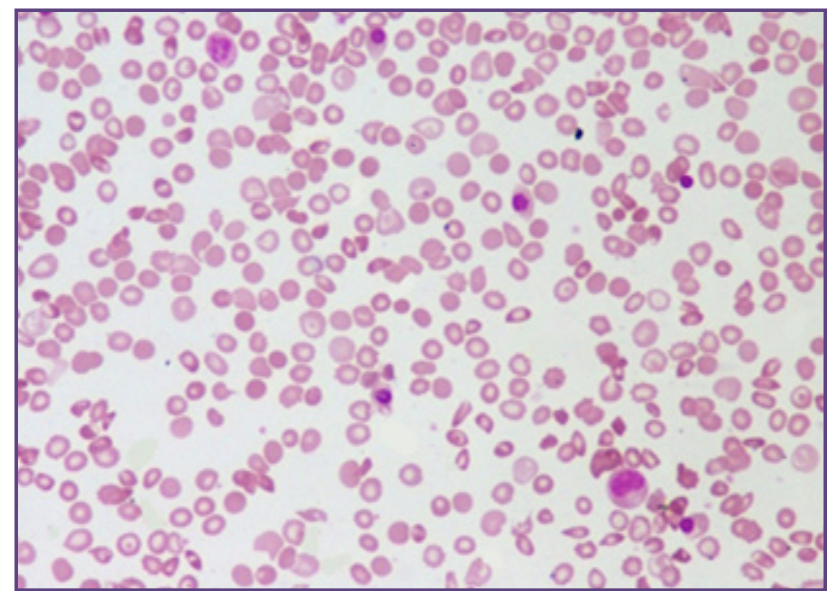

Fig. 3: PBS (400x): Thalassemia major showing few spherocytes, polychromasia, target cells, myeloid precursor and nRBCs.

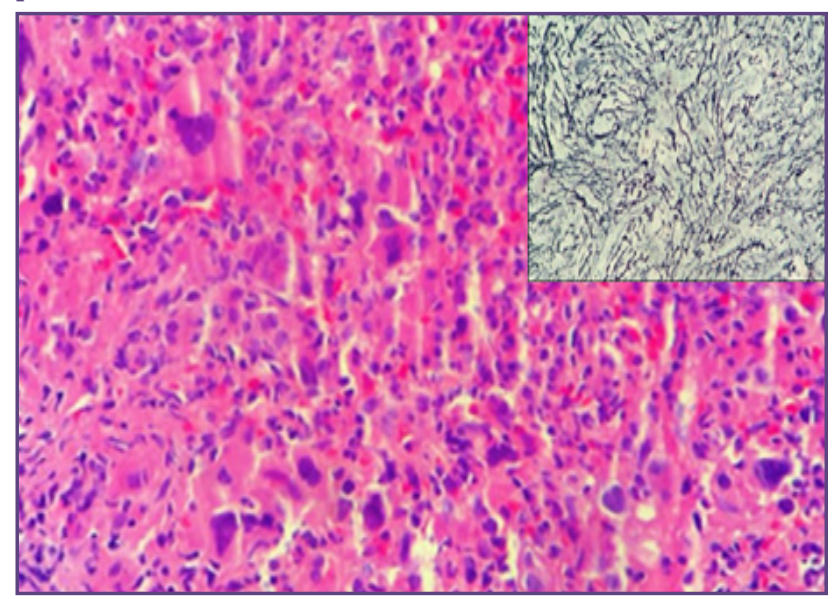

Fig. 5: BM Biopsy (100x, HE) Myelofibrosis showing cellular marrow with hyperplasia of the myeloid and megakaryocytic series with few dysplastic megakaryocytes. Inset: BM Biopsy (100x, Reticulin) Myelofibrosis showing grade 2-3 reticulin fibrosis.

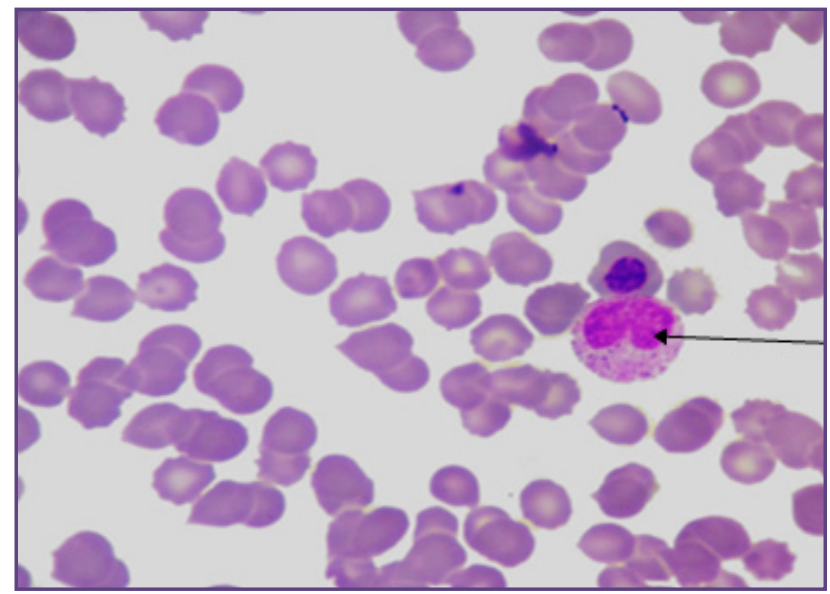

Fig. 7: PBS (1000x) case of Metastatic carcinoma showing a metamyelocyte and $\mathrm{nRBC}$.

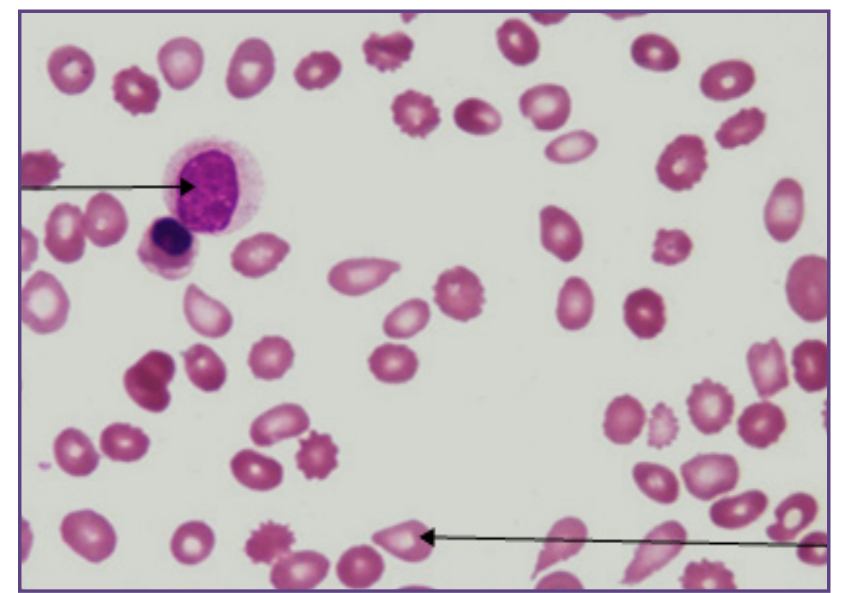

Fig. 4: PBS (1000x): Myelofibrosis showing tear drop RBCs, a myelocyte and nRBC.

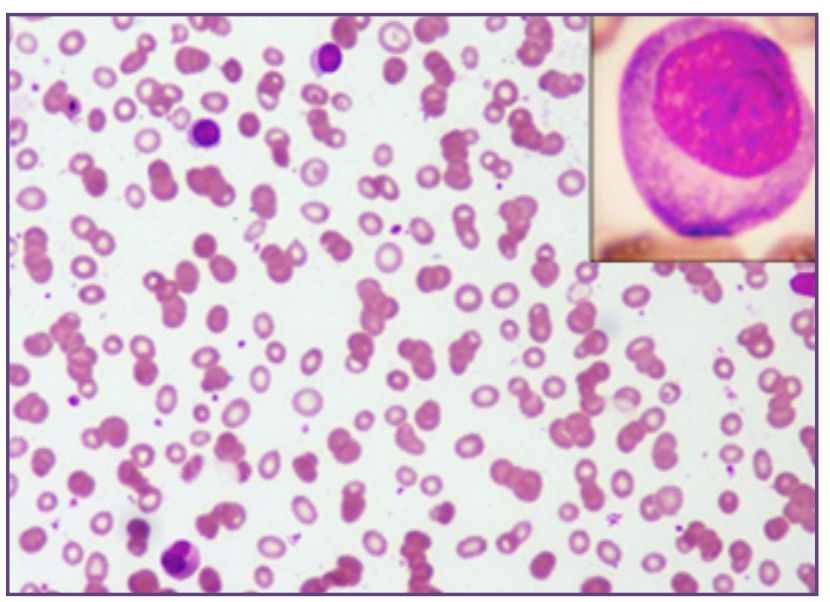

Fig. 6: PBS (400x): Multiple Myeloma showing rouleaux formation, shift to left in myeloid series and a plasma cell [Inset].

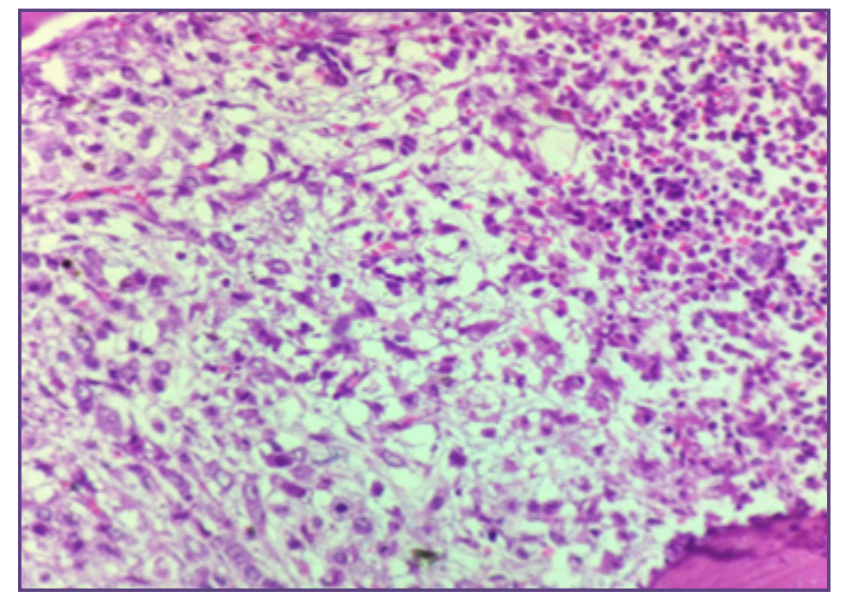

Fig. 8: BM Biopsy (400x, HE) Metastatic Carcinoma showing a tumour composed of large malignant epithelial cells. 
the cases fell in the third decade (30\%) followed by fourth decade (22\%)] and were contrasting to those by Ken Sang Lee et al. [33\% pediatric cases]. ${ }^{[4,7]}$

ii) Distribution of $\mathbf{H b}$ across cases: The hemoglobin values of cases in current study ranged from 2.5 to $12.6 \mathrm{~g} / \mathrm{dL}$ with average hemoglobin of $7.1 \mathrm{~g} / \mathrm{dL}$. The range of hemoglobin values mentioned in Vaughan's study was between 2.5 and 8.3 , with an average of 5.4 $\mathrm{g} / \mathrm{dL} \cdot{ }^{[1]}$ In the study by Retief, the range was between 2.7 and 15, with an average of $8.9 \mathrm{~g} / \mathrm{dL} .{ }^{[4]}$ Burkett et al. found the range of haemoglobin values to be in between 6.8 and $12 \mathrm{~g} / \mathrm{dL}$ with an average of $9.1 \mathrm{~g} / \mathrm{dL}$. ${ }^{[8]}$ The values in our study nearly correlates with the hemoglobin values in all these studies.

In our study, $87 \%$ of patients had hemoglobin less than $10 \mathrm{~g} / \mathrm{dL} ; 9 \%$ of patients had $\mathrm{Hb}$ values less than $4 \mathrm{~g} /$ $\mathrm{dL}$. Only one case had hemoglobin more than $12 \mathrm{~g} / \mathrm{dL}$. Maximum number of cases i.e., 78\% had hemoglobin in the range of 4 to $10 \mathrm{~g} / \mathrm{dL}$. This nearly correlates with the study by Ken Sang Lee et al. where the maximum number of cases, $69 \%$, had hemoglobin levels between 6 and $12 \mathrm{~g} / \mathrm{dL}^{[7]}$ and with the study by Burkett et al. ${ }^{[8]}$, where $76 \%$ of cases had hemoglobin in the range of 6 to $10 \mathrm{~g} / \mathrm{dL}$ and also Vaughan's study ${ }^{[1]}$, where $85 \%$ of cases had hemoglobin values ranging from 3 to $8 \mathrm{~g} / \mathrm{dL}$.

iii) Total leukocyte count [TLC] distribution: Corrected total leucocyte count in the present study ranged from $3700 / \mathrm{cmm}$ to $65800 / \mathrm{cmm}$ with an average of $20500 /$ $\mathrm{cmm}$ in our study and $79 \%$ of the cases had high count. The study by Retief et al. states the total leucocyte count ranging from $4000 / \mathrm{cmm}$ to $94000 / \mathrm{cmm}$ with an average of $16500 / \mathrm{cmm}$. Around $54 \%$ of the patients had higher leucocyte count. ${ }^{[4]}$

Regarding distribution, our study had $2 \%$ of cases with low leucocyte counts, 19\% with normal counts and
$79 \%$ with high counts. The study by Ken Sang Lee et al. had $10 \%$ of cases with low count, $39 \%$ with normal and $51 \%$ with high count ${ }^{[7]}$ and the study by Retief et al., $44 \%$ of the patients had count between $4000 / \mathrm{cmm}$ and $11000 / \mathrm{cmm}$, and remaining $56 \%$ had high count. ${ }^{[4]}$

iv) Platelet count distribution: In our study, $60 \%$ of the patients had low platelet count, normal platelet count was seen in $32 \%$ and high platelet counts in $8 \%$. This corresponds to the study by Ken Sang Lee et al. who recorded low platelet count in $60 \%$, normal in $27 \%$ and high platelet count in $13 \%{ }^{[7]}$

v) Distribution of causes of LER: In present study, hemolytic diseases (HD) was the most common (29\%) cause of the LER, followed by liver diseases (LD) $(22 \%)$ and septicemia (SP) $(18 \%)$. This distribution is different from the causes of LER mentioned in the studies by Retief et al. ${ }^{[4]}$, Vaughan et al. ${ }^{[1]}$, Burkett et $a l .^{[8]}$ and Ken Sang Lee et al. ${ }^{[7]}$ This is because of the different pool of samples for the individual study.

vi) Hemoglobin distribution among various causes of LER

In our study, average hemoglobin was lowest with megaloblastic anemia, followed by hemolytic diseases. Higher average hemoglobin values were seen with septicemia followed by liver diseases. One case of multiple myeloma also had lower hemoglobin levels. One case of autoimmune vasculitis had the highest haemoglobin level in our study (12.6 g/dL).

In Vaughan's study, lower levels of hemoglobin were seen with carcinomatosis and higher levels were seen with myelosclerosis. ${ }^{[1]}$ In Retief's study, lower haemoglobin levels were seen with carcinomatosis and hemolytic diseases and higher levels were seen with septicemia. ${ }^{[4]}$ Burkett's study associated lower levels

\begin{tabular}{|l|l|l|l|}
\hline Vaughan et al. & Retief et al. & Burkett et al. & Ken Sang Lee et al. \\
\hline Carcinomatosis, 61 & Infections, 35 & Leukemia \& Lymphoma, 31 & Leukemia \& Lymphoma, 22 \\
\hline Myelomatosis, 23 & Carcinomatosis, 11 & Myeloproliferativedisorders, 13 & Carcinomatosis, 12 \\
\hline Myelosclerosis, 8 & Hemolytic diseases, 8 & Hemolytic anemia, 9 & $\begin{array}{l}\text { Benign hematological } \\
\text { diseases, 10 }\end{array}$ \\
\hline Thalassemia, 8 & Megaloblastic anemia, 5 & Carcinomatosis, 8 & Myeloproliferative \\
& disorders, 10 & Infections, 10 \\
\hline & Myelomatosis, 5 & Megaloblastic anemia, 7 & Liver diseases, 4 \\
\hline & Myeloproliferative disorders, 5 & Infections, 7 & Multiple myeloma, 1 \\
\hline & Acute leukemia, 5 & Hemorrhage, 4 & \\
\hline
\end{tabular}


of hemoglobin with carcinomatosis, megaloblastic anemia, hemorrhage and hemolytic diseases and higher levels of hemoglobin with septicemia followed by myelosclerosis. ${ }^{[8]}$

Findings from our study correlates with that of Retief's. ${ }^{[4]}$

vii) Total leucocyte count distribution among various causes of LER

In our study, highest average corrected total count was seen with myeloproliferative disorders, followed by infections, followed by liver diseases. Lower total counts were associated with megaloblastic anemia. Our findings were in concordance with Retief's study, where higher counts were associated with myeloproliferative disorders followed by septicemia. Lower counts were associated with megaloblastic anemia, Hodgkin's lymphoma and severe sepsis. ${ }^{[4]}$

vii) Platelet count distribution among various causes of LER

In our study, higher average platelet values are associated with myeloproliferative disorders, followed by hemolytic diseases. Lowest average platelet values were seen with megaloblastic anemia, followed by infections and one case of disseminated malignancy.

Retief mentions in his study that low platelet counts were commonly associated with infections, followed by acute leukemia ${ }^{[4]}$ and this correlates with our findings.

ix) Average values of $\mathrm{Hb}$, TLC and platelet count among various causes of LER

Average hemoglobin was lowest with megaloblastic anemia, followed by hemolytic diseases. Higher average hemoglobin values were seen with septicemia. Highest average corrected total leucocyte count was seen with myeloproliferative disorders, followed by septicemia and liver diseases. Lower total leucocyte counts were associated with megaloblastic anemia. Higher average platelet values were associated with myeloproliferative disorders, followed by hemolytic diseases. Lowest average platelet values were seen with megaloblastic anemia, followed by septicemia. Megaloblastic anemia had the lowest average values of all the above three parameters.

This was in concordance with the studies by Retief ${ }^{[4]}$ and Burkett et al. ${ }^{[8]}$

x) Distribution of nucleated RBCs, Reticulocyte count and WBC precursors among various causes of LER

In the present study, $\mathrm{nRBC}$ counts were in the range of 2 to 588 with an average of 43 per 100 WBCs counted. This range in Vaughan study ${ }^{[1]}$ was in the range between 1 and 106 with an average of 36 per 100 WBCs, which was correlating with the reports of the present study. Retief ${ }^{[4]}$ reported $\mathrm{nRBC}$ count range of 1 to 19 with an average of $3 \mathrm{nRBCs}$ per $100 \mathrm{WBCs}$. In Burkett's study ${ }^{[8]}$ it was in the range of 1 to 23 with an average of 5 per 100 WBCs.

Highest reticulocyte percentage in our study was seen with hemolytic diseases, followed by megaloblastic anemia. The causes associated with lower reticulocyte count in descending order of frequency were myeloproliferative disorders, infections and liver diseases, and one case of disseminated malignancy. This is in concordance with other studies in literature. Vaughan associated higher reticulocyte percentages with thalassemia and lower percentages with myelosclerosis. ${ }^{[1]}$ Burkett also associated high reticulocyte percentages with hemolytic diseases followed by cases with hypoxia, while he also mentions one case of leukemia ${ }^{[4]}$, classified under 'other leukemias' to have a high reticulocyte count of $31 \%$, which probably might be erythroleukemia. Low average percentages of reticulocyte counts were mentioned to be seen with multiple myeloma, infections and acute leukemia. ${ }^{[8]}$

In our study, counts of leucocyte precursors (metamyelocytes, myelocytes, promyelocytes and myeloblasts) varied from 3 to 44 percentage, with an average of 11.Vaughan mentions a range of 1 to 12 precursors in 200 cell WBC differential. ${ }^{[1]}$ Retief mentions precursors count to range from 2 to 69 with average of 9.1. In Burkett's study, range was from 1 to 95 with average of $13 .^{[8]}$

Average number of WBC precursors in the present study correlate with the average in all these studies. Also in our study, highest number and high average number of WBC precursors were seen with myeloproliferative diseases and this was followed by septicemia. Lower numbers were seen with multiple myeloma, disseminated malignancy and hemolytic diseases. This is in concordance with the findings from Retief et al. study in which highest number of WBC precursors were seen with myelofibrosis, followed by chronic myelogenous leukemia, followed by infection. ${ }^{[4]}$ Also it matches with the Burkett's study, where higher numbers were seen with acute leukemia, followed by chronic myelogenous leukemia, followed by myelofibrosis and infections. Lower numbers were associated with multiple myeloma, hemolytic diseases and megaloblastic anemia. ${ }^{[8]}$ 
xi) Mean corpuscular volume (MCV) and Red blood cell distribution width (RDW-SD) among various causes of LER

$\mathrm{MCV}$ values in our study ranged from 53.2 to $124 \mathrm{fL}$, with an average of $85 \mathrm{fL}$. RDW-SD values ranged from 11.3 to 40, with an average of 23.In Vaughan's study, MCV was measured only in 4 cases, and it ranged from 83.6 to 109 , with an average of $87.5 \%{ }^{\left[{ }^{[1]}\right.}$

Higher values of mean corpuscular volume (MCV) in our study were seen with megaloblastic anemia, highest MCV noted being $124.2 \mathrm{fL}$ with an average of 103 fL.Average low values were seen with hemolytic diseases. Higher values of red cell distribution width (RDW - SD) were seen with myeloproliferative disorders, followed by hemolytic diseases.

MCV and RDW values weren't studied in association with leucoerythroblastosis in literature, yet Vaughan mentions a case with high MCV (109 fL) in a splenectomised case of myelosclerosis, attributing the presence of macrocytosis to splenomegaly. ${ }^{[1]}$

\section{Conclusion}

With current study, it is evident that leucoerythroblastic reaction can be seen in a variety of conditions apart from carcinomatosis, as believed once [9].These various etiologies should be considered or ruled out when this picture is encountered on peripheral blood smear. Detailed analysis of hematological parameters and examination of peripheral blood smear for morphology of RBCs, WBCs and platelets give a clue to the etiology. Work up with further investigations is required for confirmation of the etiology. Very limited literature is available on this topic and hence this publication will enrich the evidence available.

\section{Limitations}

The present study was conducted in a single institute. Multicentre studies in similar context would shed more light on the subject. We have collected data from only one institute; therefore, population is relatively homogenous.

\section{References}

1. Vaughan JM, Oxon DM. Leuco-erythroblasticanemia. Journal of Pathology and Bacteriology. 1936; 42 (3): 541564.

2. Byard RW, Bormanis J, Jones TG. Leukoerythroblastosis: a much maligned phenomenon?. CMAJ. 1987; 137(3):191.

3. Lazar GS, Forman SJ: Leukoerythroblastic reaction in Still disease in an adult. West J Med 1972;131:152-155

4. Retief F. Leuco-erythroblastosis in the adult. The Lancet. 1964; 283(7334):639-642.

5. Clifford GO. The clinical significance of leukoerythroblastic anemia. The Medical Clinics of North America. 1966; 50(3):779.

6. Canbolat Ayhan A, Timur C, Ayhan Y, Kes G. Leukoerythroblastosis Mimicking Leukemia: A case report. Iran J Pediatr. 2014 Jun;24(3):332-3.

7. Ken Sang Lee et al. Clinical significance of Leucoerythroblastosis. Korean Journal of Internal Medicine. 1982; 25(7):694-697.

8. Burkett LL, Cox ML, Fields ML. Leukoerythroblastosis in the adult. American Journal of Clinical Pathology. 1965; 44(5):494-498.

9. Delsol G, Guiu-Godfrin B et al. Leukoerythroblastosis and cancer frequency, prognosis, and physio pathologic significance. Cancer. 1979; 44:1009-1013.

*Corresponding author:

Dr Daksha Prabhat, Professor, Department of Pathology, 1st Floor, Dean Office Building, Seth G.S. Medical College and KEM Hospital, Parel, Mumbai. 400012 INDIA

Phone: +919322270087

Email: dakshaprabhat@gmail.com

Financial or other Competing Interests: None. 